\title{
The Relationship between Flux Coefficient and Entrainment Ratio in Density Currents
}

\author{
MATHEW WELLS \\ University of Toronto, Toronto, Ontario, Canada \\ Claudia Cenedese \\ Woods Hole Oceanographic Institution, Woods Hole, Massachusetts \\ C. P. CAulfield \\ BP Institute and Department of Applied Mathematics and Theoretical Physics, University of Cambridge, \\ Cambridge, United Kingdom
}

(Manuscript received 21 January 2009, in final form 26 July 2010)

\begin{abstract}
The authors explore the theoretical and empirical relationship between the nonlocal quantities of the entrainment ratio $E$, the appropriately depth- and time-averaged flux coefficient $\bar{\Gamma}$, and the bulk Froude number $\operatorname{Fr}_{o}$ in density currents. The main theoretical result is that $E=0.125 \bar{\Gamma} \operatorname{Fr}_{o}^{2}\left(C_{U}^{3} / C_{L}\right) / \cos \theta$, where $\theta$ is the angle of the slope over which the density current flows, $C_{L}$ is the ratio the turbulent length scale to the depth of the density current, and $C_{U}$ is the ratio of the turbulent velocity scale to the mean velocity of the density current. In the case of high bulk Froude numbers $\bar{\Gamma} \sim \operatorname{Fr}_{o}^{-2}$ and $\left(C_{U}^{3} / C_{L}\right)=C_{\epsilon} \sim 1$, so $E \sim 0.1$, consistent with observations of a constant entrainment ratio in unstratified jets and weakly stratified plumes. For bulk Froude numbers close to one, $\bar{\Gamma}$ is constant and has a value in the range of $0.1-0.3$, which means that $E \sim \mathrm{Fr}_{o}^{2}$, again in agreement with observations and previous experiments. For bulk Froude numbers less than one, $\bar{\Gamma}$ decreases rapidly with bulk Froude number, explaining the sudden decrease in entrainment ratios that has been observed in all field and experimental observations.
\end{abstract}

\section{Introduction}

The dynamics of density currents are of fundamental importance for understanding the transport and mixing properties of dense waters, such as the Antarctic Bottom Water or North Atlantic Deep Water. These water masses form at high latitudes and eventually fill up most of the deep regions of the world's ocean basins. The final properties of these water masses are largely determined by the amount of interfacial mixing or "entrainment" that occurs between these density currents and the lighter overlying water masses. A popular approach to modeling the dynamics of oceanic density currents has been through the development of "stream tube" models, whereby the Coriolis force, buoyancy

Corresponding author address: Mathew Wells, Department of Physical and Environmental Sciences, University of Toronto, 1265 Military Trail, Toronto ON M1C 1A4, Canada.

E-mail: wells@utsc.utoronto.ca forces, bottom drag, and increasingly complex topography can be incorporated to predict the behavior of density currents (Price and Barringer 1994). One of the central assumptions in these models is the "entrainment hypothesis" of Taylor (1945), for a review see Turner (1986), whereby the rate at which the fluid in a density current turbulently entrains surrounding ambient fluid is assumed to be linearly proportional to the mean downstream velocity $U$, which is often expressed in terms of an entrainment ratio defined as

$$
E=w_{e} / U,
$$

where $w_{e}$ is the entrainment velocity normal to the current, as shown in Fig. 1.

Although the magnitude of the entrainment ratio is of central importance to understanding how many deepwater masses form (Price and Barringer 1994; Baines 2001; Papadakis et al. 2003; Wells and Wettlaufer 2007; Wells and Nadarajah 2009; Legg et al. 2009), there has 


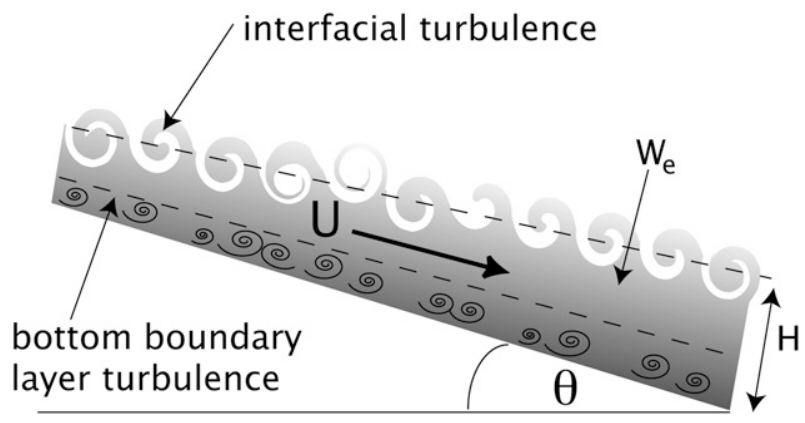

FIG. 1. A sketch of the entrainment velocity $w_{e}$ into a dense current of mean velocity $U$. The current has a thickness $H$ and flows down a slope of angle $\theta$. Gravity $g$ acts at an angle $\theta$ to the entrainment velocity $w_{e}$. The entrainment velocity is controlled by turbulence generated at the sheared upper interface. The thickness of this interface is generally a large fraction of the total depth of the current. In the interior of the dense current the density gradient is homogenized by turbulence generated at the bottom boundary layer.

not previously been any unifying theory to predict either the magnitude of $E$ or to determine how $E$ depends upon the stability of the flow. The results to date are based on empirical observations from laboratory and field studies (Cenedese and Adduce 2010). For a vertical plume or an unstratified jet, the entrainment ratio is found to be approximately constant, $\sim 0.1$ (Taylor 1945; Ellison and Turner 1959; Turner 1986). In stratified density currents flowing down slopes, the entrainment ratio decreases as the stability of the current increases (Ellison and Turner 1959; Turner 1986). In Fig. 2, we have plotted a graph of available observations of entrainment ratio versus the bulk Froude number, defined as

$$
\operatorname{Fr}_{o} \equiv \frac{U}{\sqrt{G^{\prime} H \cos \theta}}
$$

where $U$ is the characteristic along-stream mean velocity of the density current, $H$ is the characteristic thickness of the current, $\theta$ is the angle of the slope, and $G^{\prime}$ is the reduced gravity. Here, we determine an appropriate definition for the current depth $H$ from the properties of the density distribution. These time- and depth-averaged characteristic quantities of the flow can be defined (e.g., following Arneborg et al. 2007) by considering the three integrals:

$$
\begin{gathered}
G^{\prime} H=I_{1}=\frac{1}{T_{t}} \int_{0}^{T_{t}} \int_{o}^{\infty} \frac{\bar{\rho}-\rho_{o}}{\rho_{o}} g d z d t, \\
\frac{1}{2} G^{\prime} H^{2}=I_{2}=\frac{1}{T_{t}} \int_{0}^{T_{t}} \int_{o}^{\infty} \frac{\bar{\rho}-\rho_{o}}{\rho_{o}} g z d z d t,
\end{gathered}
$$

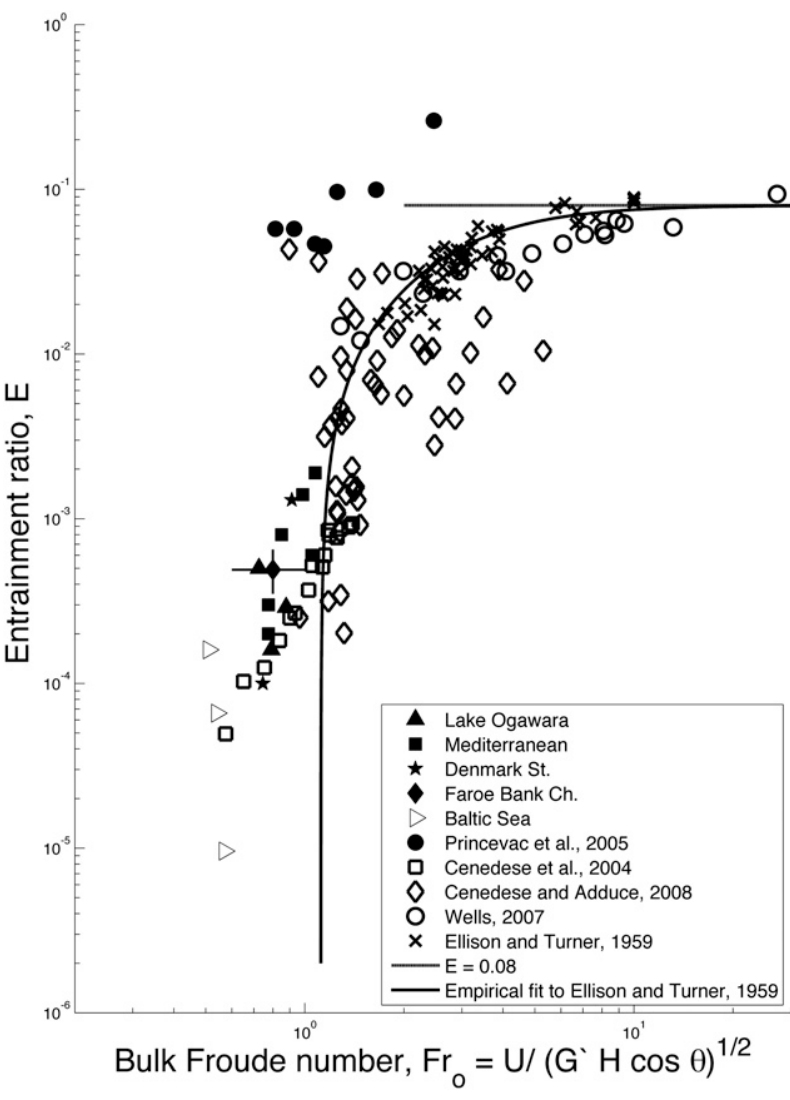

FIG. 2. Measurements of entrainment ratio $E$ plotted as a function of the bulk Froude number. The data comes from field and laboratory experiments over a wide range of Reynolds numbers. The oceanic data span five location sites: the Mediterranean overflow (Baringer and Price 1997), Denmark Strait (Girton and Sanford 2003), Faroe Bank Strait (Mauritzen et al. 2005), the Baltic Sea (Arneborg et al. 2007), and Lake Ogawara (Dallimore et al. 2001). The laboratory experiments include both nonrotating (Ellison and Turner 1959) and rotating (Cenedese et al. 2004; Cenedese and Adduce 2008; Wells 2007) experiments on densitydriven currents descending a slope. The entrainment parameterization of Turner (1986) is plotted as a solid line and asymptotes to $E=0.08$ (dashed line) at large $\mathrm{Fr}_{o}$.

$$
U H=I_{3}=\frac{1}{T_{t}} \int_{0}^{T_{t}} \int_{o}^{\infty} \bar{u} d z d t
$$

and

$$
H=\frac{2 I_{2}}{I_{1}} ; \quad U=\frac{I_{3} I_{1}}{2 I_{2}} ; \quad G^{\prime}=\frac{I_{1}^{2}}{2 I_{2}}
$$

where $g$ is gravity, $\bar{\rho}$ is the depth-dependent density (with the overline denoting an appropriate horizontal average), $\rho_{\mathrm{o}}$ is the ambient density, and $T_{t}$ is a sufficiently long time scale over which to average to remove small-scale random turbulent fluctuations-substantially longer than any typical eddy turnover time of the flow 
yet still short compared to time scales over which the bulk flow evolves. Implicit in this definition is an appropriate averaging across stream for the entire current. Therefore, in principle the quantities on the left-hand side of Eqs. (3)-(5) could vary on a "slow" time scale either to reflect the accumulated effect of the turbulence averaged over the "fast" time scale $T_{t}$ or external variations in forcing. In practice however, owing to the relative paucity of time-dependent experimental or observational measurements, the quantities on the left-hand side should be considered as typical values for any particular flow. Hereafter, when we refer to averaged quantities, we mean the time and depth averaging used in Eqs. (3)-(5).

In Fig. 2, one can see that the entrainment ratio increases with increasing bulk Froude number and that there appears to be a transition in the slope around $\mathrm{Fr}_{o}=1$. For $\mathrm{Fr}_{o}>1$ the entrainment ratio is similar to $E \sim \mathrm{Fr}_{o}^{2}$ or $E \sim \mathrm{Fr}_{o}^{1}$, whereas there is a much steeper slope for $\mathrm{Fr}_{o}<1$. We would like to determine the functional relationship between the entrainment ratio and the bulk Froude number. One commonly used parameterization of the entrainment ratio data obtained in the laboratory by Ellison and Turner (1959) was proposed by Turner $(1986)$ as $E=\left(0.08-0.1 \mathrm{Ri}_{o}\right) /\left(1+5 \mathrm{Ri}_{o}\right)$ for $\mathrm{Ri}_{o}<0.8$. This empirical curve is plotted in Fig. 2 with $\mathrm{Fr}_{o}=\mathrm{Ri}_{o}^{-1 / 2}$ and is clearly a poor fit to all the data with $\mathrm{Fr}_{o}<1$.

As the entrainment ratio and bulk Froude number are both depth-averaged quantities of a density current, we will focus on the depth-averaged values of dissipation, buoyancy flux, and flux coefficient and will consider that the entrainment process is inherently shear driven. There are two primary sources of turbulence in a density current, namely the interfacial entrainment at the upper boundary and turbulence generated by drag at the bottom boundary, as illustrated in Fig. 1. These two sources of turbulence both contribute to the overall momentum budget through the drag coefficient $C_{D}$ and the entrainment ratio $E$ (Baringer and Price 1997), but only the turbulence generated at the upper interface is important in determining the value of the entrainment ratio. When profiles of dissipation are measured in oceanographic overflows, there is usually a minimum in dissipation at the level of the maximum flow velocity (Johnson et al. 1994; Peters and Johns 2005; Umlauf and Arneborg 2009) indicating that turbulence generated at the bottom boundary cannot be the principal source of entrainment of overlying fluid into the flow (as assumed in Canuto et al. 2005), although it can act to homogenize the interior of the density current. Observations in a shallow saline density current by Dallimore et al. (2001) suggested that, at least in their flow, the bottom boundary layers could merge with the interface so that, for very shallow low Froude number flows, this bottom-generated turbulence might be important for mixing. However, since the entrainment is primarily driven by mixing at the upper interface, it seems appropriate to study this process in terms of a shear-driven mixing parameterization, as pointed out by Jackson et al. (2008). The mean profile of both velocity and density in the interfacial region is nearly linear (Ellison and Turner 1959; Peters and Johns 2005), so it makes sense to look at the entrainment in terms of a bulk Froude number based on the averaged properties.

The purpose of this paper is to determine how to relate the empirical observations of averaged flux coefficient in a stratified flow to the entrainment ratio of stratified density currents. A fundamental issue is how to parameterize the various characteristics of the turbulent flow crucial to mixing processes in terms of external, measurable mean quantities of the flow under consideration. In section 2, we propose an approach to calculating a flux coefficient of density currents, using the averaged buoyancy flux into a density current and the scaling of the averaged dissipation rate of the interfacial region. The resulting expression for the flux coefficient is then found to be a function of the entrainment ratio. In section 3, we will use this relationship and empirical observations of the flux coefficient to predict how the entrainment ratio of a density current changes with the bulk Froude number of the flow. These predictions are then compared with laboratory and field data. In section 4 , we draw some brief conclusions.

\section{The relationship between entrainment ratio and flux coefficient as a function of bulk Froude number}

The dependence of the entrainment ratio on the bulk Froude number must be related to how efficiently turbulence can convert the kinetic energy of the flow into irreversible mixing within the interfacial region. There have been several studies (Linden 1979, 1980; Ivey and Imberger 1991; Fernando 1991; Strang and Fernando 2001a,b) that have explored the relationship between the efficiency of mixing and the strength of the stratification. The overall efficiency of a flow in converting kinetic energy into potential energy may be defined (as in Ivey and Imberger 1991) in terms of a flux Richardson number as

$$
\mathrm{Ri}_{F}=\frac{-\mathcal{B}}{-\mathcal{B}+\mathcal{E}},
$$

where $\mathcal{B}$ and $\mathcal{E}$ are appropriate spatial and temporal averages of the turbulent buoyancy flux and dissipation rate, defined as 


$$
\mathcal{B}=-\frac{1}{T_{t} H} \int_{0}^{T_{t}} \int_{\delta}^{\infty} \frac{g}{\rho_{0}} \overline{\rho^{\prime} u_{3}^{\prime}} d z d t=-\frac{1}{T_{t} H} \int_{0}^{T_{t}} \int_{\delta}^{\infty} \bar{b} d z d t
$$

and

$$
\begin{aligned}
& \mathcal{E}=\frac{1}{T_{t} H} \int_{0}^{T_{t}} \int_{\delta}^{\infty} \overline{\frac{\nu}{2}} \overline{\left.\frac{\partial u_{i}^{\prime}}{\partial x_{j}}+\frac{\partial u_{j}^{\prime}}{\partial x_{i}}\right)\left(\frac{\partial u_{i}^{\prime}}{\partial x_{j}}+\frac{\partial u_{j}^{\prime}}{\partial x_{i}}\right)} d z d t \\
& =-\frac{1}{T_{t} H} \int_{0}^{T_{t}} \int_{\delta}^{\infty} \bar{\epsilon} d z d t .
\end{aligned}
$$

In these expressions, $b$ and $\epsilon$ are the conventional spatially and temporally varying definitions of the turbulent buoyancy flux and turbulent dissipation rate, and the overline denotes an appropriate horizontal average. Primed variables denote perturbations away from the horizontally averaged flow quantities; $u_{3}^{\prime}$ is the fluctuation velocity in the vertical $z$ direction, with the Einstein summation convention being used in the definition of the dissipation rate and the current depth $H$ being as defined in (6). Since we are primarily concerned about the dissipation in the neighborhood of the density interface, we exclude the bottom boundary layer thickness $\delta$ from the integrals (8) and (9). Thus, in these definitions (as discussed above) we assume that the behavior at the lower solid boundary does not dominate the calculation of these averaged quantities, so the turbulence (leading both to entrainment and dissipation) in the interfacial region is assumed to be nontrivial, a situation that we expect to occur in many circumstances of interest.

Embedded in the expression (7) for the flux Richardson number (for a fuller discussion, see Strang and Fernando 2001a) is that the flow is quasi stationary, with turbulent production being balanced by the irreversible losses from the kinetic energy reservoir. This means that the expression may be interpreted as an "efficiency," with the denominator quantifying the total loss rate from the mean kinetic energy reservoir (corresponding to the turbulent production in steady state) and the numerator quantifying the rate of stratified mixing, leading to increases in potential energy. The quantity $\mathrm{Ri}_{F}$ is usually defined (as here) as a single average value for a stratified turbulent flow, rather than varying spatially (Linden 1979; Strang and Fernando 2001a). An important further subtlety is that the time scale $T_{t}$ must be chosen so that the net effect of the fluctuating buoyancy flux $b$ is to increase the potential energy of the flow through irreversible mixing. Choosing $T_{t}$ to be long compared to typical eddy turn over times is likely to filter out periods of the flow evolution when potential energy is being converted into kinetic energy through stirring processes and, thus, for the buoyancy flux to reflect only irreversible mixing (for a fuller discussion, see Peltier and Caulfield 2003).

A closely related parameter used in the oceanographic community is the quantity $\bar{\Gamma}$, which we shall refer to as the flux coefficient, following the usage in, for example, Smyth et al. (2001). The flux coefficient is the ratio of the buoyancy flux to the dissipation rate, so we define it as

$$
\bar{\Gamma} \equiv \frac{-\mathcal{B}}{\mathcal{E}} .
$$

We have used the overline notation to make it clear that this quantity is spatially and temporally averaged to be a characteristic quantity for the entire flow. As originally discussed by Osborn (1980; see also Ivey et al. 2008), the flux coefficient is conventionally used as a way to determine the (usually harder to measure or estimate) pointwise value of the buoyancy flux $b$ from the (usually easier to measure or estimate) pointwise value of the dissipation rate $\epsilon$ that is,

$$
-b=\Gamma \epsilon .
$$

Expressed in this way, it is then clear why (10) is the natural definition for an appropriately averaged version of this flux coefficient, particularly since it is not guaranteed at all points in space and time that the buoyancy flux is negative. Thus, the local effect of the turbulence on the stable stratification is to increase the potential energy, which is of course the expected net effect of turbulent mixing in a stably stratified flow over sufficiently long time scales. As noted above, it is entirely possible for transient "stirring" events to have positive buoyancy flux, as elevated dense parcels of fluid convert their potential energy into kinetic energy, whereas dissipation must always be positive definite. In such scenarios, very common in the evolution of shear-driven flow instabilities such as Kelvin-Helmholtz billows (see Peltier and Caulfield 2003), the definition (11) is problematic, so we will always use the definition (10) for the (averaged) flux coefficient.

Although the quantity defined in (10) is often referred to as the mixing efficiency in the oceanographic literature, we will try to avoid this usage. The flux Richardson number $\mathrm{Ri}_{F}$ as defined in (7) is more appropriately referred to as a mixing efficiency and, by definition, is guaranteed to be less than one. On the other hand, considering (10), there is no fundamental reason why the integrated average value of the dissipation rate over some time interval must always be greater than the integrated value of the buoyancy flux.

However, in realistic flows that are largely statically stable, it has been common to assume that $\bar{\Gamma} \leq 0.2$ for 
a)

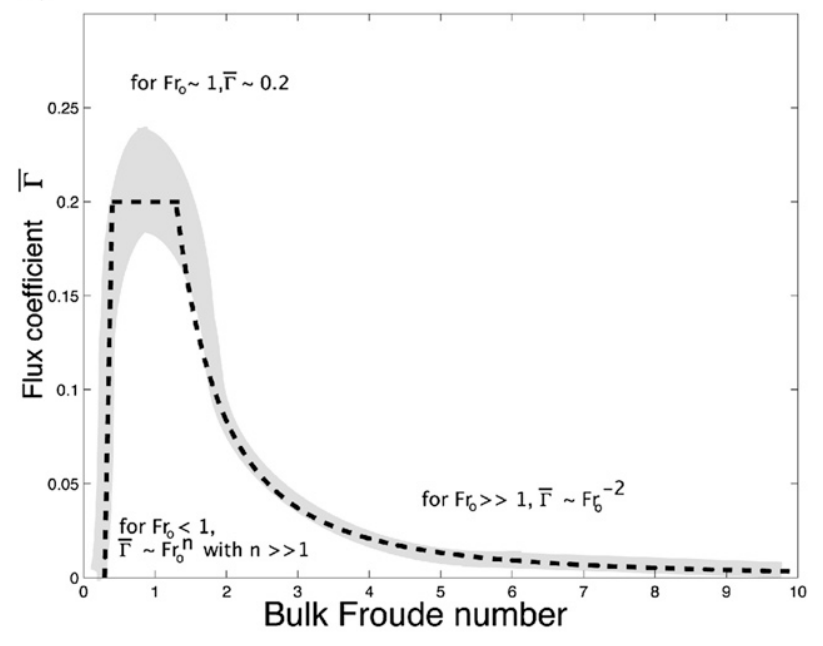

b)

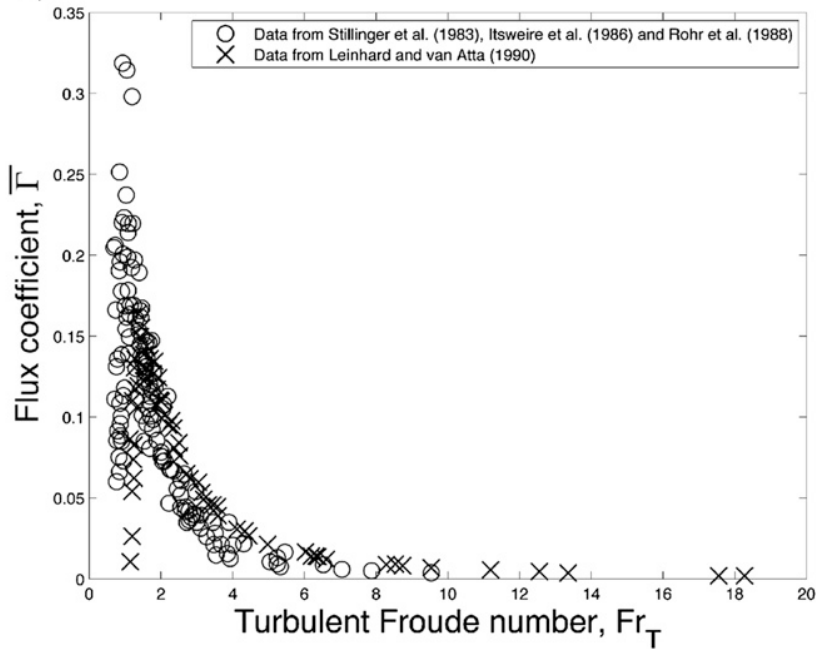

FIG. 3. (a) Sketch of the relationship between the flux coefficient and bulk Froude number: the shaded area illustrates the approximate behavior found in the experiments of Linden (1980), Ivey and Imberger (1991), and Strang and Fernando (2001a,b). For very high and very low bulk Froude numbers $\bar{\Gamma} \rightarrow 0$; for $\operatorname{Fr}_{o} \sim 1$ there is a maximum value, $0.15<\bar{\Gamma}<0.25$. Some experiments indicate that for high bulk Froude numbers $\bar{\Gamma} \sim \mathrm{Fr}_{o}^{-2}$, while at low bulk Froude numbers, $\bar{\Gamma} \rightarrow 0$ at some finite value $\mathrm{Fr}_{o}>0$. (b) Experimental data of the flux coefficient vs turbulent Froude number, data from Fig. 6a of Ivey and Imberger (1991). This graph shows similar behavior to the experiments of Linden (1980) and Strang and Fernando (2001a,b).

most wave-breaking and shear-driven mixing events, so measurements of dissipation in the ocean can be converted to the vertical eddy diffusivity via $K_{\rho}=\bar{\Gamma} \mathcal{E} / N^{2}$ (Osborn 1980). We note in this relationship that the arguments in Osborn (1980) imply the flux coefficient $\bar{\Gamma}$ is only meaningful for the time- and space- (or ensemble) averaged equations. The spatially and temporally averaged flux coefficient $\bar{\Gamma}$ is related to the flux Richardson number (7) by

$$
\bar{\Gamma}=\frac{\mathrm{Ri}_{F}}{1-\mathrm{Ri}_{F}},
$$

So, in the limits where $\mathrm{Ri}_{F}$ goes to zero, $\bar{\Gamma}$ also approaches zero (Ivey and Imberger 1991). For intermediate bulk Froude numbers $\bar{\Gamma}$ will have a similar peak to $\mathrm{Ri}_{F}$.

In Fig. 3a, we have summarized the results from the experiments of Linden (1980), Ivey and Imberger (1991), and Strang and Fernando (2001a,b). An example of one of these datasets from Ivey and Imberger is plotted in Fig. 3b. All of the previous experiments show the same general behavior, with a peak flux coefficient between 0.1 and 0.3 that occurs near a bulk Froude $\mathrm{Fr}_{o} \sim O(1)$ or a turbulent Froude number $\mathrm{Fr}_{T} \sim O(1)$ (where the turbulent Froude number is defined as $\operatorname{Fr}_{T}=U_{T} / N L_{T}$, with $L_{T}$ and $U_{T}$ the rms turbulent length and velocity scales within a stratified flow with larger-scale buoyancy frequency $N$ ). As the bulk Froude number becomes smaller than one, the turbulence is strongly damped and the flux coefficient rapidly decreases, essentially because of the suppression of strong overturning mixing events. As the bulk Froude number becomes larger than one, the flux coefficient again decreases as there is less density gradient to mix.

There is some (albeit inconclusive) indication in the various experiments that, for high bulk Froude numbers $\bar{\Gamma} \sim \mathrm{Fr}_{o}^{-2}$ and at low bulk Froude numbers, the flux coefficient $\bar{\Gamma}$ may go to zero at some finite value of $\mathrm{Fr}_{o}$. Similar comments on the variation of the flux coefficient with the flow stability were made by Ivey et al. (2008), where they noted that the flux Richardson number $\mathrm{Ri}_{F} \rightarrow 0$ as the turbulent activity parameter or buoyancy Reynolds number $\mathrm{Re}_{b} \rightarrow 1$ and as $\mathrm{Re}_{b} \rightarrow$ $10^{5}$, where $\mathrm{Re}_{b}$ is defined as

$$
\operatorname{Re}_{b} \equiv \frac{\epsilon}{\nu N^{2}}
$$

They observed that the flux Richardson number is maximal with $\mathrm{Ri}_{F} \sim 0.25$ between these two extremes when $\operatorname{Re}_{b} \sim O\left(10^{2}\right)$. Shih et al. (2005) showed that $\mathrm{Re}_{b} \sim \mathrm{Re}_{T} / \mathrm{Ri}_{o}$ where $\mathrm{Re}_{T}=U_{T} L_{T} / \nu$ so that the flux Richardson number $\mathrm{Ri}_{F}$ and, hence, flux coefficient depends on both mean and turbulent flow properties. While it is clear that the average measures of the flow stability, such as the bulk Froude and Richardson numbers, cannot be directly related to the turbulent Froude 
number or the buoyancy Reynolds number, the main result we will use for this paper is that the flux coefficient has a maximum value for $\mathrm{Fr}_{o} \sim 1$ and that $\bar{\Gamma}$ decreases as $\mathrm{Fr}_{o} \rightarrow 0$ and $\mathrm{Fr}_{o} \rightarrow \infty$.

A common scaling argument in the experimental literature [see Christodoulou (1986) or Fernando (1991) for a detailed review] is that the entrainment ratio $E \propto \mathrm{Ri}_{o}^{-1}$ for at least intermediate values of bulk Richardson number $\mathrm{Ri}_{o}$, which is equivalent to $E \propto \mathrm{Fr}_{o}^{2}$. As noted by Kato and Phillips (1969), this implies that the rate of change of the potential energy of the system is proportional to the kinetic energy flux in the vicinity of an interface over which the mixing is occurring. In other words, as the turbulent motions inject kinetic energy to the interface, a certain amount of the energy leads to mixing. As discussed in detail by Crapper and Linden (1974) and demonstrated experimentally recently by Zellouf et al. (2005), this $\mathrm{Ri}_{o}^{-1}-\mathrm{Fr}_{o}^{2}$ power law behavior occurs for flows with sufficiently high Peclet numbers (i.e., $\mathrm{Pe}=\operatorname{RePr}$, where $\operatorname{Pr}=\nu / \kappa$ is the Prandtl number, $\operatorname{Re}=$ $U L / \nu$ is the Reynolds number, $\kappa$ is the thermal diffusivity, $\nu$ is the viscosity), and the appropriate length scale $L$ is chosen so that diffusive processes do not play a significant dynamical role.

Such a situation would be expected to occur in an oceanic flow. However, it is important to appreciate that the particular grid turbulence-driven forcing used in these studies is qualitatively different from the sheardriven mixing expected in density currents, so such power law behavior is not guaranteed to occur. At high values of the bulk Richardson number $\mathrm{Ri}_{o}$ (low $\mathrm{Fr}_{o}$ ) there is also experimental evidence of a change in the relationship so that $E \propto \mathrm{Ri}_{o}^{-3 / 2}\left(E \propto \mathrm{Fr}_{o}^{3}\right)$ [see Linden (1973) and the review of Christodoulou (1986)], which is attributed to a decrease in the overturn time scale of vortices at the interface compared to the increasing characteristic response time of the highly stratified interface. Furthermore, the experiments quoted in Ivey and Imberger (1991) show that, for a fixed value of the turbulent Froude number, the flux coefficient increases with the Reynolds number of the flow, as has been found both numerically by Riley and de Bruyn Kops (2003) and experimentally by Prastowo et al. (2008). Numerical simulations of stratified shear flows (for a review, see Peltier and Caulfield 2003) show a peak in the flux coefficient of $\bar{\Gamma} \sim 0.2$ and also that $\bar{\Gamma}$ increases with low $\mathrm{Ri}_{o}$. Indeed, such values are relatively high compared to experimental measurements of mixing in stratified flows where $\bar{\Gamma}$ is typically observed to be substantially less than one, such as in the grid-mixing experiments of Rehmann and Koseff (2004) and the vertical rod mixing experiments of Holford and Linden (1999) or Martin and Rehmann (2006) in which maximum values were $\bar{\Gamma} \simeq 0.05-0.1$.
We now seek to determine a quantitative relationship between the (averaged) flux coefficient $\bar{\Gamma}$ and the entrainment ratio in a density current. Once we have done this, we will be able to use the empirical observations of averaged dissipation and flux coefficient from sheardriven flows to predict the functional form of the entrainment ratio. To do this, we need an expression for the averaged buoyancy flux $\mathcal{B}$, as defined in (8), through the interface of a density current and an estimate of the average dissipation rate of energy in the interface $\mathcal{E}$. Then, we can evaluate the averaged flux coefficient using $\bar{\Gamma}$, as defined in (10). As already noted, it is important to define an average buoyancy flux $\mathcal{B}$ for the density current as a whole, as local values of the pointwisebuoyancy flux $b$ vary throughout the flow, starting with a zero value at the bed, increasing approximately linearly toward a maximum in the entrainment layer, and then rapidly approaching zero above the density current. Under the assumption that the entrainment velocity $w_{e}$ must be related to the turbulent velocity fluctuations $u_{3}^{\prime}$, (1), (3), and (8) can be related (Strang and Fernando 2001a) to obtain

$$
-\mathcal{B}=G^{\prime} U E \cos \theta .
$$

We note that, in the gravity current literature, there is often confusion between this type of definition of the entrainment ratio $E$ based on the buoyancy flux as in (14) and an alternate version based on volume conservation [e.g., $E=1 / U d / d x(U H)$, where $x$ is downstream coordinate, Turner 1973], which is what is often thought of as entrainment. Although the two are equivalent in an idealized slab model, the relationship between the integral-based definitions is not clear and depends on the specific shapes of the density, velocity, and buoyancy flux profiles.

Equation (14) for the buoyancy flux is then combined with $\bar{\Gamma}=-\mathcal{B} / \overline{\mathcal{E}}$ to give a relationship between the entrainment ratio $E$ and the flux coefficient $\bar{\Gamma}$ (analogously to the approach of Osborn 1980),

$$
E=\frac{\mathcal{E}}{G^{\prime} U} \frac{\bar{\Gamma}}{\cos \theta}
$$

The central aim of this paper is to identify appropriate scalings for each of these terms and, thus, obtain a relationship between $E$ and $\bar{\Gamma}$, which in general varies with the overall strength of the stratification. Intuitively Eq. (15) has the right form in that, when $\mathrm{Fr}_{o}$ is very small, where $\bar{\Gamma}$ goes to zero, then $E$ goes to zero as expected. For the case of large $\mathrm{Fr}_{o}$, we expect that the average dissipation rate $\mathcal{E}$ should increase with the velocity of the flow so that an increase in $\mathcal{E}$ may balance the decrease in 
a)

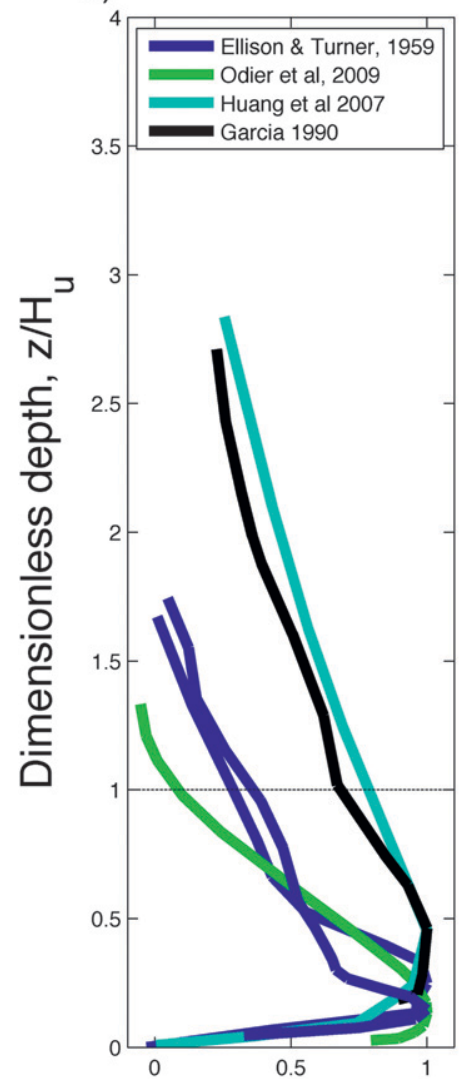

b)

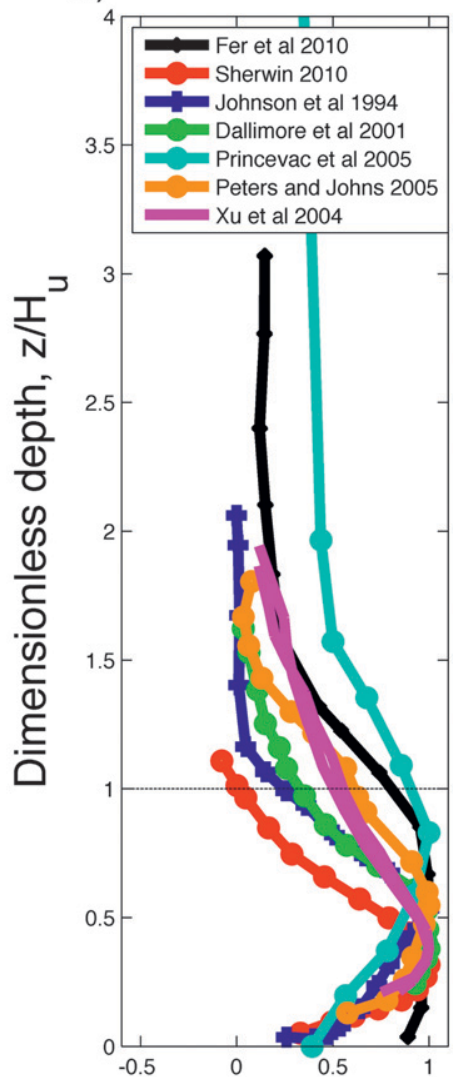

c)

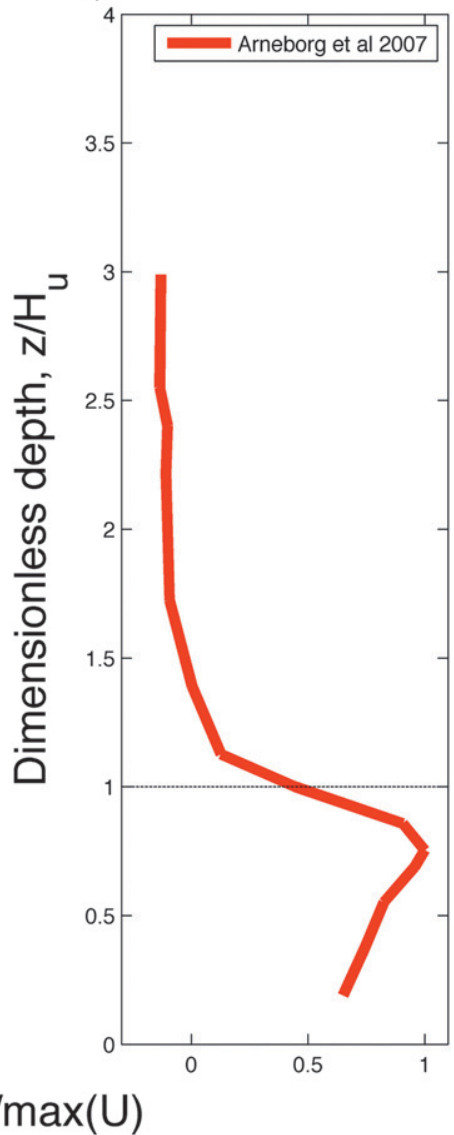

FIG. 4. Velocity profiles plotted from (a) laboratory scale experiments, (b) oceanographic scale gravity currents, and (c) the shallow density current flowing into the Baltic Sea. Depth is normalized by the depth of the current $H$, defined as $H_{u}=\left(\int u d z\right)^{2} / \int u^{2} d z$ (Ellison and Turner 1959). Thickness of the interface might reasonably be defined as the depth over which the velocity changes from $25 \%$ to $75 \%$ of the maximum value. In every case, except for (c), the interface thickness is comparable to the depth of the current (data from Ellison and Turner 1959; Garcia 1990; Johnson et al. 1994; Dallimore et al. 2001; Xu et al. 2004; Peters and Johns 2005; Huang et al. 2007; Arneborg et al. 2007; Odier et al. 2009; Fer et al. 2010; Sherwin 2010).

$\bar{\Gamma}$, resulting in the constant entrainment ratio $E \sim 0.1$ found for unstratified jets and plumes by Turner (1986).

We would like to predict the functional form of $E$ based on knowledge of $\bar{\Gamma}$ and average dissipation rate $\mathcal{E}$. Experiments on stratified mixing reported in Ivey and Imberger (1991) found that the dissipation rate is related to the turbulent velocity scales $U_{T}$ and turbulent length scales $L_{T}$ as

$$
\mathcal{E}=\frac{1}{8}\left(U_{T}^{3} / L_{T}\right)
$$

This definition is implicitly a spatially and temporally averaged quantity as it is using the rms values of turbulent length and velocity scales.

From (15) and (16), it is thus necessary to identify appropriate scalings for $L_{T}$ and $U_{T}$. The characteristic turbulence scale $L_{T}$ is due to overturnings in the interface and would be expected to scale with the interface thickness. Of course, this begs the question of how to scale the interface. For high bulk Froude number flows in laboratory experiments and in most oceanic density currents, the interface region is comparable to the total thickness of the flow (Figs. 4a and 4b). In these plots, the velocity is normalized by the maximum velocity and the depth is normalized by a characteristic depth of the current $H_{u}$, determined from the characteristics of the velocity distribution and defined as (following Ellison and Turner 1959)

$$
H_{u}=\frac{\left(\frac{1}{T_{t}} \int_{0}^{T_{t}} \int_{0}^{\infty} \bar{u} d z d t\right)^{2}}{\frac{1}{T_{t}} \int_{0}^{T_{t}} \int_{0}^{\infty} \overline{u^{2}} d z d t} .
$$


As in (3)-(5), the overbar denotes an appropriate horizontal average, and the time scale $T_{t}$ is sufficiently long to integrate over characteristic turbulent fluctuations. Typically, $H_{u} \simeq H$ [as defined in (6)] in situations where both the velocity and density distribution are known. The depth defined by $H_{u}$ is a more generally applicable definition for oceanic flows since the presence of background stratification means that it is often not clear how to determine the background density to evaluate the term $\bar{\rho}-\rho_{o}$ using field measurements of the density profile. We have not included the corresponding density profile shapes, but note that in general the location and thickness of the density interface coincides closely with the velocity interface.

Though common, there are certainly flows that are qualitatively different from those shown in Figs. 4a,b. In contrast to the broad interfacial region seen in laboratory-scale flows and most oceanic flows, the velocity profile shown in Fig. $4 \mathrm{c}$ from the overflow in the Baltic Sea has a much sharper velocity interface (Arneborg et al. 2007; Umlauf and Arneborg 2009). The density profile is not shown but is also much sharper than seen in most other density currents. This particular density current in the Baltic Sea has a very low bulk Froude number flow, as shown in Fig. 2, and also has the lowest observed entrainment ratios. Umlauf and Arneborg note that, in this particular current where the interfacial mixing is relatively weak, interfacial entrainment may not be the dominant mixing mechanism. Instead, they identified two other stronger mechanisms. The presence of a transverse circulation and a lateral density gradient resulted in strongly enhanced mixing on the southern side of the current. Also, near the thin edges of the current, turbulence generated in the bottom boundary layer could directly influence mixing at the upper boundary. However, since this particular low Froude number density current seems to be qualitatively different from the large range of other data presented in Fig. 4, we will assume that for most density currents the interface thickness scales with the depth $H$ of the density current, as defined in (6), although we will discuss situations where the interface is substantially smaller than the depth of the current.

Indeed, a key related issue is the associated vertical length scale over which the velocity shear is significant and over which nontrivial vertical turbulent velocities are maintained, which naturally will then determine the length scale over which entrainment occurs. When $\mathrm{Fr}_{o}$ is large, this length scale is likely to be comparable with the depth of the flow, while for lower $\mathrm{Fr}_{o}$ vertical motions are inevitably reduced given the increase in the strength of the stratification (Linden 1979; Smyth and Moum 2000a,b; Billant and Chomaz 2001; Brethouwer et al. 2007).
The data shown in Fig. 4 suggest that for almost all cases (except perhaps for the lowest Froude numbers) the interface is of the same scale as the depth of the current so that $L_{T}=C_{L} H$. The largest value of $L_{T}$ is when overturning eddies fill the whole of the interface thickness so that, in general, $C_{L} \leq 1$. The rms turbulence fluctuations $U_{T}$ are the velocity scale at which energy is input into mixing and thus should scale as $U$, so we can assume that $U_{T}=C_{U} U$, where $C_{U} \leq 1$. If stratification is very strong, as is the case for $\mathrm{Fr}_{o}<1$, then the turbulent length scale will be reduced, as well as the turbulent velocity scales so that both $C_{L} \ll 1$ and $C_{U} \ll 1$. Hence, the appropriately averaged dissipation rate $\epsilon$ (dominated by its values within the interface) should scale like

$$
\begin{aligned}
\mathcal{E} & =\frac{U^{3}}{8 H}\left(\frac{C_{U}^{3}}{C_{L}}\right)=C_{\epsilon} \frac{U^{3}}{8 H}, \quad C_{L}=\frac{L_{T}}{H} ; \\
C_{U} & =\frac{U_{T}}{U} ; \quad C_{\epsilon}=\frac{C_{U}^{3}}{C_{L}},
\end{aligned}
$$

where in general $C_{L}, C_{U}$, and hence $C_{\epsilon}$ may well depend on external flow parameters, such as the Froude number.

Oceanographic observations of turbulent microstructure suggest that, when $\mathrm{Fr}_{o}<1$, the mean dissipation rate $\mathcal{E}$ in the interface of a density current is typically on the order of $\mathcal{E}=10^{-6} \mathrm{~W} \mathrm{~kg}^{-1}$. For example, the observations of dissipation rate in the Mediterranean overflow by Johnson et al. (1994) find average values on the order of $\mathcal{E}=10^{-6}$ to $10^{-7} \mathrm{~W} \mathrm{~kg}^{-1}$ in the 70 -m thick interface. The total flow has a thickness of $H=150 \mathrm{~m}$ and a mean velocity of $U=0.8 \mathrm{~m} \mathrm{~s}^{-1}$, suggesting that, for this overflow, $C_{\epsilon} \sim O\left(10^{-2}\right)$. There are similar estimates of average dissipation rates in the 120-m thick interface of the Red Sea overflow reported by Peters and Johns (2005). This overflow has a thickness of $H=250 \mathrm{~m}$ and a mean velocity of $U=0.4 \mathrm{~m} \mathrm{~s}^{-1}$, suggesting similar values of $C_{\epsilon} \sim O\left(10^{-2}\right)$. The bulk Froude number of both overflows is between 0.7 and 1 .

When $\mathrm{Fr}_{o}>1$, we expect the damping effect of stratification to become weaker, thus allowing the eddies to become both larger (increasing $C_{L}$ ) and more energetic (increasing $C_{U}$ ) in a way that increases the ratio $C_{\epsilon}$. For instance, List (1982) reports values in jets and plumes of $\sqrt{U_{T}^{2}} / U \sim 0.3$, suggesting a value of $C_{U}=0.3$. Similar observations of velocity fluctuations where $U_{T} / U \sim 0.25$ were reported for a density current with a mean value of $\mathrm{Fr}_{o}=2.6$ (Odier et al. 2009). In this density current, the mixing length scale was $O(0.01 \mathrm{~m})$ in the $0.08-\mathrm{m}$ deep flow, suggesting $C_{L}=1 / 8$ so that $C_{\epsilon}=0.18-0.3$. The turbulence in the density current of Odier et al. was dominated by interfacial mixing and the averaged dissipation rate measured was $\mathcal{E} \sim O\left(10^{-4}\right) \mathrm{W} \mathrm{kg}^{-1}$. Such a dissipation rate would be consistent with using (18) with 
$C_{\epsilon} \sim 0.1-0.5$, that is, similar to the estimates made from the length and velocity scales in the interfacial region.

If we combine (18) with (15), we obtain an important result of this paper, an expression for the entrainment ratio in terms of the bulk Froude number and the average flux coefficient,

$$
E=\frac{C_{\epsilon}}{8} \frac{\bar{\Gamma} \mathrm{Fr}_{o}^{2}}{\cos \theta} .
$$

We note that a similar equation to (19) was derived by Linden (1979, his Eq. 4.1) in the context of mixing by grid stirring at a stratified interface. Furthermore, this expression makes it clear that there are several essential issues, which determine how $E$ depends on $\mathrm{Fr}_{o}$. Most obviously, it is reasonable to suppose that the (averaged) flux coefficient $\bar{\Gamma}$ depends on $\mathrm{Fr}_{o}$. More subtly, both $C_{L}$ (the ratio of the turbulent length scale over which the entrainment is occurring to the depth of the current) and $C_{U}$ (the ratio of the characteristic turbulent velocity length scale to the mean velocity $U$ of the current) may in general be functions of the overall stratification, that is, on $\mathrm{Fr}_{o}$ ), so the expression for $E$ may depend in a more complicated fashion on $\mathrm{Fr}_{o}$. To investigate this, we consider three different bulk Froude number limits where we can make useful scaling arguments to determine the functional form of $E\left(\mathrm{Fr}_{o}\right)$, paying particular attention to how $\bar{\Gamma}$ and the constants $C_{L}$ and $C_{U}$ (and hence their natural combination $C_{\epsilon}$ ) are likely to vary from one regime to the next.

\section{a. The limit of high bulk Froude number}

The first case to consider is that of high bulk Froude number where stratification is expected to be relatively unimportant. Numerous authors (Linden 1979, 1980; Strang and Fernando 2001a,b) have shown that at low flow stability the flux coefficient can be expressed as a decreasing function of the flow stability; that is, $\bar{\Gamma} \sim \mathrm{Fr}_{o}^{-2}$. A similar result was found by Ivey and Imberger (1991), who summarized results of previous experimental studies (Stillinger et al. 1983; Itsweire et al. 1986; Rohr et al. 1988; Lienhard and van Atta 1990) where for high turbulent Froude numbers the flux coefficient was found to be $\bar{\Gamma}=(1 / 3) \mathrm{Fr}_{T}^{-2}$.

As the interfacial region of most density currents has a well defined density and velocity gradient that is on average linear, it is sensible to compare entrainment dynamics of density currents to previous experimental determinations of the flux coefficient where a linear density and velocity gradient was used, as in Ivey and Imberger (1991) and Strang and Fernando (2001a,b). If we assume that $\bar{\Gamma}=1 /\left(a \mathrm{Fr}_{o}^{2}\right)$ for $a>1$, then using (19) the entrainment ratio can be written as

$$
E=\frac{C_{\epsilon}}{8 a \cos \theta} .
$$

The important result here is that the entrainment ratio is independent of the bulk Froude number when $\mathrm{Fr}_{o} \gg 1$, provided of course that $C_{\epsilon}$ does not in turn depend on $\mathrm{Fr}_{o}$ in this limit. The results of Odier et al. (2009) implied that for $\mathrm{Fr}_{o}>1$ a realistic estimate of the ratio $C_{\epsilon} \sim 0.5$. It seems reasonable to identify the turbulent Froude number with the bulk Froude number in these weakly stratified flows where $C_{\epsilon}$ is $O(1)$, thus suggesting that the characteristic length and velocity scales of the turbulence are of the same order as the equivalent scales of the bulk flow. Then using the Ivey and Imberger (1991) result that $\bar{\Gamma}=(1 / 3) \mathrm{Fr}_{T}^{-2}$ would imply that the constant $a=3$ so that $E=0.04$ in close agreement with the empirical observations where $E=0.07-0.1$ (Turner 1986). In this high bulk Froude number limit the density currents are dominated by momentum rather than buoyancy forces, so a constant value of $E \sim 0.1$ is found, as is the case for all unstratified jets and plumes (Ellison and Turner 1959; Turner 1986).

\section{b. The regime of intermediate bulk Froude number}

The second case to consider is for intermediate bulk Froude numbers around $\mathrm{Fr}_{o}=1$, where the flux coefficient has a maximum value of around $\bar{\Gamma} \sim 0.25$ (consistent with the evidence from shear-driven turbulence simulations as reviewed by Peltier and Caulfield 2003), and can be considered constant, as illustrated in Fig. 3. The peak value of flux Richardson number reported by Ivey and Imberger (1991) is in the range $\mathrm{Ri}_{F}=$ $0.12-0.24$ so that $\bar{\Gamma}=0.13-0.31$. It is expected that the value of maximum flux coefficient is a function of Reynolds number, with higher Reynolds numbers having higher values of $\bar{\Gamma}$ but that, above a certain "critical" Reynolds number, the value of $\bar{\Gamma}$ remains constant, as shown in the numerical simulations of Riley and de Bruyn Kops (2003). If we assume for simplicity that for $\mathrm{Fr}_{o} \sim O(1)$, the flux coefficient $\bar{\Gamma}$ is constant and equal to 0.2 , then (19) becomes

$$
E=0.025 \frac{C_{\epsilon} \mathrm{Fr}_{o}^{2}}{\cos \theta}, \text { for } \operatorname{Fr}_{o} \sim 1
$$

An upper bound on the entrainment ratio is when $C_{L} \sim$ $C_{U} \sim 1$ (and hence $\mathrm{C}_{\epsilon}=1$ ), with the characteristic scales of the turbulence corresponding to the scales of the outer, bulk flow, where we would obtain

$$
E=0.025 \frac{\mathrm{Fr}_{o}^{2}}{\cos \theta}, \text { for } \mathrm{Fr}_{o} \sim 1
$$




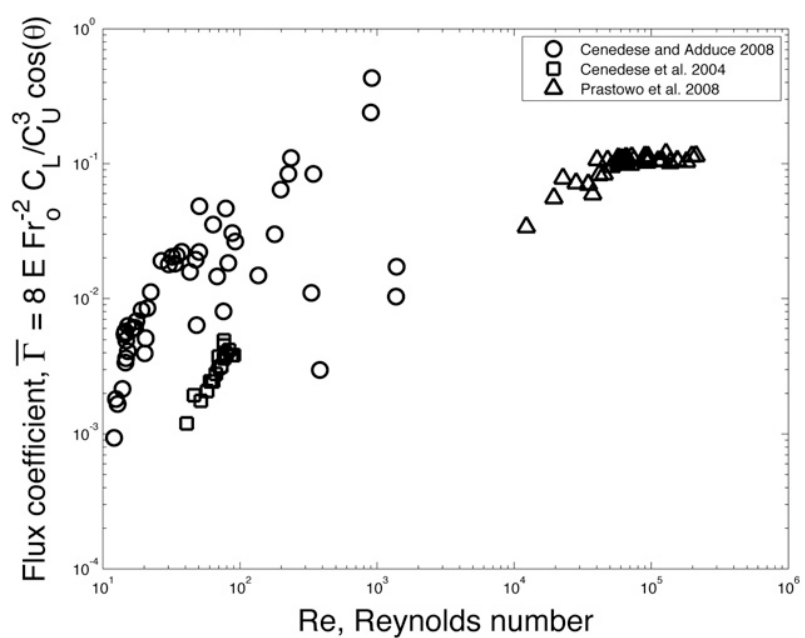

FIG. 5. Experimental observations suggest that the flux coefficient is a strong function of the Reynolds number. Here, we plot the estimated flux coefficient from the experiments of Cenedese and Adduce (2008) and Cenedese et al. (2004), along with the reported flux coefficient from Prastowo et al. (2008). Although there is some scatter in these data, the flux coefficient is clearly increasing with the Reynolds number.

which appears to describe the high values of entrainment ratio seen in the atmospheric katabatic flow data of Princevac et al. (2005).

A reasonable lower bound on the entrainment ratio is when $C_{\epsilon}=10^{-2}$, as appears to be the case in oceanic density currents, that is, where the velocity and length scales of the turbulence are $O(10 \%)$ of the (external) current values. In this case with $\bar{\Gamma}=0.2$, we would obtain

$$
E=2.5 \times 10^{-4} \frac{\mathrm{Fr}_{o}^{2}}{\cos \theta}, \text { for } \operatorname{Fr}_{o} \sim 1
$$

which predicts the right order of magnitude of $E \sim 10^{-4}$ for the oceanic data shown in Fig. 2 for $\mathrm{Fr}_{o} \sim 1$.

We note that the numerical factor of 0.025 in this equation is obtained assuming a maximum flux coefficient of $\bar{\Gamma}=0.2$, following Osborn (1980), which may be correct only for high Reynolds number flows dominated by shear-induced mixing. There is experimental evidence that the flux coefficient and entrainment ratios increases with Reynolds number (Ivey and Imberger 1991; Riley and de Bruyn Kops 2003; Prastowo et al. 2008; Cenedese and Adduce 2008) so that experiments at low Reynolds number flows are expected to have a factor less than 0.025 in (21). Prastowo et al. (2008) found that $\bar{\Gamma}$ increases with Reynolds number until $\mathrm{Re}=$ $7 \times 10^{4}$ after which $\bar{\Gamma}$ has a constant, albeit substantially smaller value of 0.11 (Fig. 5). The numerical simulations of Riley and de Bruyn Kops (2003, see their Fig. 19) show a somewhat larger value of $\bar{\Gamma} \sim 0.4$ for large Re, consistent with the estimate generated by a theoretical upper bound on the long-time-averaged buoyancy flux discussed in Caulfield et al. (2004).

In Fig. 5, we show how $\bar{\Gamma}$ estimated from laboratory experiments appears to vary with bulk Reynolds number, using experimental data of Cenedese et al. (2004), Cenedese and Adduce (2008), and Prastowo et al. (2008). The data show a trend with the predicted values of $\bar{\Gamma}$ increasing with increasing Reynolds number. In Fig. 5, the flux coefficient was estimated from the reported entrainment ratios in Fig. 2 using $\bar{\Gamma}=8 E \mathrm{Fr}_{o}^{-2} \cos \theta$, obtained from (19) assuming the simplest case of $C_{\epsilon}=1$.

As already noted above, the relationship (21) suggesting that $E \sim \mathrm{Fr}_{o}^{2}$ is consistent with observations of Ellison and Turner (1959). If we express $E \sim \mathrm{Fr}_{o}^{2}$ in terms of a bulk Richardson number, then we get $E \sim$ $\mathrm{Ri}_{o}^{-1}$, as predicted by Eq. (9.1.4) in Turner (1973), and consistently with Kato and Phillips (1969), the grid turbulence measurements of Zellouf et al. (2005), and, indeed, a wide range of studies as reviewed in Christodoulou (1986) and Fernando (1991). For $1<\mathrm{Fr}_{o}<10$ the entrainment ratio has been previously empirically described as $E \sim \mathrm{Fr}_{o}$ by Price and Barringer (1994). The observations of the entrainment ratio in Fig. 2 suggest that there is a gradual transition in slope or change in power law as a function of bulk Froude number. Hence, the scaling that $E \sim \mathrm{Fr}_{o}$ from Price and Barringer (1994) may represent an intermediate regime between the constant $E=0.1$ obtained for high bulk Froude numbers and the scaling that $E \sim \mathrm{Fr}_{o}^{2}$, which we have just discussed.

\section{c. The limit of low bulk Froude number}

For bulk Froude numbers less than one the flux coefficient decreases with bulk Froude number and possibly may go to zero at some finite value of the bulk Froude number. The experimental data of Linden (1980), Ivey and Imberger (1991), and Strang and Fernando (2001a,b) do not give a clear indication of how $\bar{\Gamma}$ scales with bulk Froude number in this lower limit, but for the sake of argument let us assume that $\bar{\Gamma} \sim \operatorname{Fr}_{o}^{n}$, where $n \gg 1$. If we assume that the dissipation scales as (18), then using $\bar{\Gamma} \sim \mathrm{Fr}_{o}^{n}$, and (19) implies that $E \sim \operatorname{Fr}_{o}^{n+2}$, consistent with the steeper dependence of $E$ on $\mathrm{Fr}_{o}$ observed in Fig. 2 . This argument is also based on the assumption that $C_{\epsilon}$ does not depend on $\mathrm{Fr}_{o}$. It is entirely plausible that the power law for the decay in $E$ at small $\mathrm{Fr}_{o}$ could be even greater, because the stabilizing effect of stratification will reduce $C_{\epsilon}$ provided $C_{U}$ (the ratio $U_{T} / U$ ) decays at least as fast as $C_{L}^{1 / 3}$ (the ratio $L_{T} / L$ ); that is, the stratification reduces the characteristic velocity 
scale sufficiently fast compared to the characteristic length scale of turbulence.

Continued mixing at low Froude number is consistent with intermittent turbulent events associated with these inevitable patches of low $\mathrm{Ri}$, those intermittent patches of low Ri now often being referred to in the fluid dynamical community as stratified turbulence. The scaling issue of the suppression of vertical motions by stratification has been considered by Billant and Chomaz (2001) and is at the heart of the developing understanding of the flow regime sometimes referred to as stratified turbulence, where the flow is very strongly stratified but is also at sufficiently high Reynolds number so that the buoyancy Reynolds number as defined in (13) is large, even when $N$ is large. [Here, to reiterate, $N$ is some appropriate (but not highly localized) buoyancy frequency.] We note that the oceanographic microstructure community often refers to the same process as intermittent turbulence (Gregg 1987). The inviscid scaling balance of Billant and Chomaz (2001) has now been demonstrated numerically (see Brethouwer et al. 2007) and plausibly identified in observations (Riley and Lindborg 2008). Essentially, if $\mathrm{Re}_{b} \gg 1$, inevitably regions of the flow are characterized by very low local gradient Richardson number at very high Reynolds number, even for asymptotically low values of the bulk Froude number. Hence, the flow is in the bulk extremely strongly stratified. Because of this combination of low gradient Richardson number and high Reynolds number, these patches of the flow become (intermittently) sufficiently unstable to shear instabilities for nontrivial but highly localized turbulent mixing events to occur. Therefore, although the stratification is very strong (in the sense that the overall bulk Froude number $\mathrm{Fr}_{o} \ll 1$ ), mixing is expected to continue, albeit at a markedly reduced rate.

In this regime, there is a self-similar scaling such that the characteristic vertical length scale $l_{v}$ of the flow scales like $U / N$. The vertical length scale $l_{v}$ is naturally the scale over which the entrainment occurs, so $l_{v} \sim L_{T}$. Therefore, in this regime, $C_{L}=L_{T} / H \simeq \mathrm{Fr}_{o}$. Similarly, as discussed by Billant and Chomaz (2001), the vertical velocity scale (clearly the characteristic scale of the turbulent entrainment processes) scales as

$$
w \sim U_{T} \sim \operatorname{Fr}_{o}\left(\frac{H}{l_{h}}\right) U,
$$

where $l_{h}$ is the characteristic horizontal length scale of the flow. Therefore, (24) implies that the ratio $C_{\epsilon}=$ $\operatorname{Fr}_{o}^{2}\left(H / l_{h}\right)^{3}$. In section 2, the observations of dissipation in the oceanic overflows where $0.5<\mathrm{Fr}_{o}<1$ were approximately $C_{\epsilon} \sim O\left(10^{-2}\right)$. If the ratio of the depth of

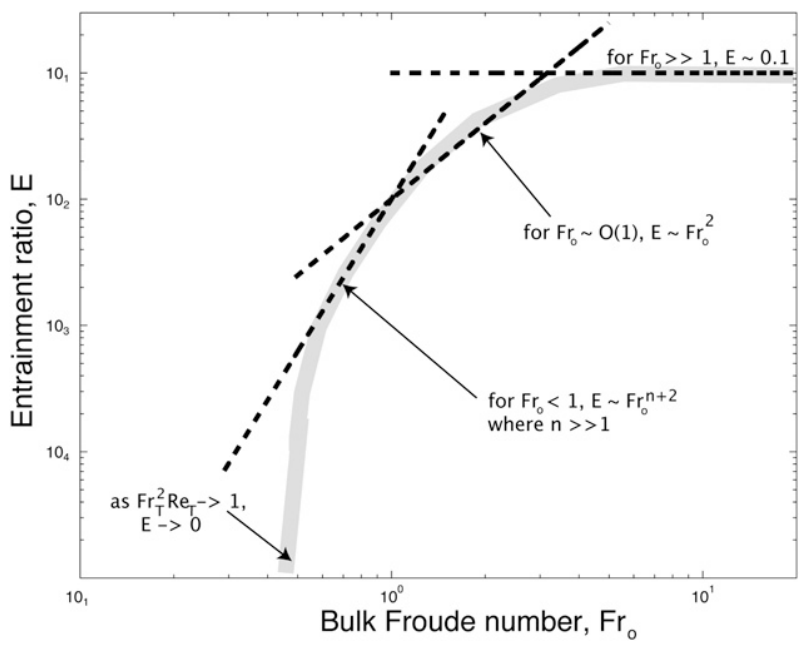

FIG. 6. A sketch of how the entrainment ratio is expected to scale with bulk Froude number. For very high bulk Froude number $E$ will be constant. For bulk Froude number $\mathrm{Fr}_{o} \sim O(1)$, we expect $E \sim \mathrm{Fr}_{o}^{2}$. For $\mathrm{Fr}_{o}<1$, there will be a steeper slope such as $E \sim \mathrm{Fr}_{o}^{4}$ Below a critical value of $\mathrm{Fr}_{o}$, where $\mathrm{Fr}_{I}^{2} \mathrm{Re}_{T} \rightarrow 1$, the entrainment ratio may drop to zero if the flux coefficient becomes zero. In this limit, the background turbulence in the ocean, for example, due to breaking internal waves, will be dominant in driving any weak mixing across the surface of the density current.

the density current to the characteristic horizontal turbulent length scale is on the order of $0.1<H / l_{h}<0.3$, then $\operatorname{Fr}_{o}^{2}\left(H / l_{h}\right)^{3}=O\left(10^{-2}\right)$. Numerical simulations by Brethouwer et al. (2007) have found ratios of $l_{h} / l_{v}$ in the range from 10 to 100 , which together with $l_{h} \sim 3-10 \mathrm{H}$ are consistent with $C_{L}=l_{v} / H \ll 1$, as might be expected for $\mathrm{Fr}_{o}<1$. Therefore, in this case,

$$
E=\frac{1}{8} \frac{\bar{\Gamma}}{\cos \theta} \operatorname{Fr}_{o}^{4}\left(\frac{H}{l_{h}}\right)^{3},
$$

so we expect the entrainment ratio to have a steep dependence on the bulk Froude number $\mathrm{Fr}_{o}$, as seen in Fig. 2 . This therefore is a prediction for a high $\mathrm{Re}_{b}$ intermittent turbulence regime of continued (though substantially reduced in quantity) mixing at strong stratifications.

On the other hand, if the flux coefficient becomes zero at some finite value of bulk Froude number, Eq. (15) implies that the entrainment ratio will also equal zero at the same value of bulk Froude number. This is expected to occur as viscosity becomes important or equivalently when the buoyancy Reynolds number $\operatorname{Re}_{b}=\epsilon / \nu N^{2} \rightarrow 1$ (Ivey et al. 2008), so the flow is not at sufficiently high Reynolds number for the intermittent turbulence regime discussed above to be sustainable. Using the definitions of the turbulent Froude number and the turbulent Reynolds number, Ivey et al. (2008) show that this limit is equivalent to the $\operatorname{Fr}_{T}^{2} \operatorname{Re}_{T} \rightarrow 1$, as indicated in Fig. 6 . 

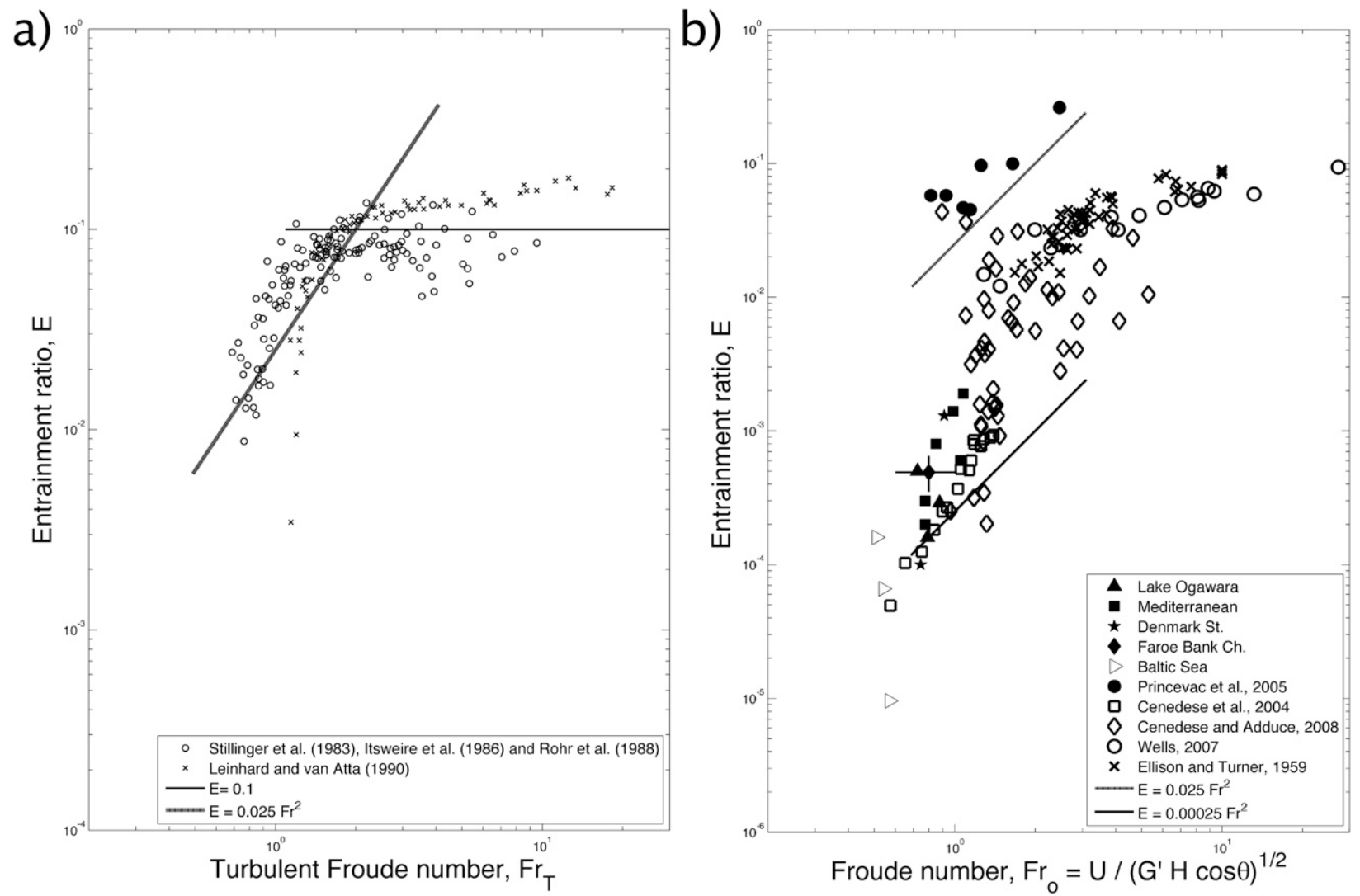

FIG. 7. (a) The entrainment ratio is estimated, using (19), from the values of flux coefficient from data in Ivey and Imberger (1991) shown in Fig. 3b. The data shows the regime at high $\mathrm{Fr}_{T}$, where $E \sim 0.1$, and the regime at intermediate $\mathrm{Fr}_{T}$, where $E \sim 0.025 \mathrm{Fr}_{T}^{2}$, similar to the predictions made for bulk Froude number. (b) Observations of entrainment ratios plotted in Fig. 2 are compared to the scaling of (19), (22), and (23) that $E \sim \mathrm{Fr}_{o}^{2}$.

It is important to appreciate that this complete suppression of mixing is also consistent with the steep decrease in entrainment ratio shown in Fig. 2.

However, there is a marked paucity of experimental data at low bulk Froude numbers, especially where the buoyancy Reynolds number is much larger than one. This means we are unable to estimate the functional form of $E$ as a function of $\mathrm{Fr}_{o}$ for the intermittent turbulence regime or, indeed, determine if the flux coefficient does in fact go to zero for some finite $\mathrm{Fr}_{o}$, thus distinguishing between the proposed intermittent turbulence and the viscously affected regimes. [Nevertheless, we can still calculate the entrainment ratio via (19).] This particular issue is undoubtedly highly relevant to the flow shown in Fig. 4c. Clearly, this is an area of research warranting further investigation since real geophysically relevant flows are quite possibly (for more discussion, see Riley and Lindborg 2008) in the intermittent turbulence regime with high $\mathrm{Re}$ and small $\mathrm{Fr}_{o}$. A summary of the scaling of $E$ for different bulk Froude number regimes is illustrated in Fig. 6. We have overlaid the expected slopes over a gray line that represents the data in Fig. 3a.

\section{Estimating an entrainment ratio from observed flux coefficients}

We will now use observations of the flux coefficient, discussed in Ivey and Imberger (1991), to estimate the entrainment ratios using Eq. (19), which will illustrate the different power-law scalings of $E$. The data reported by Ivey and Imberger are described by a turbulent Froude number rather than a bulk Froude number, so there are problems in making a quantitative comparison between $\mathrm{Fr}_{T}$ with $\mathrm{Fr}_{o}$ in (19). This is inherently related to how the quantities $C_{L}$ and $C_{U}$ are parameterized since, from (2) and (18),

$$
\mathrm{Fr}_{T}=\frac{U_{T}}{N L_{T}}=\operatorname{Fr}_{o} \frac{C_{U}}{C_{L}}
$$

With this qualification, we use Eq. (19) and $C_{\epsilon}=C_{U}^{3} / C_{L}=$ 1 to convert the flux coefficients, shown in Fig. 3b, into 

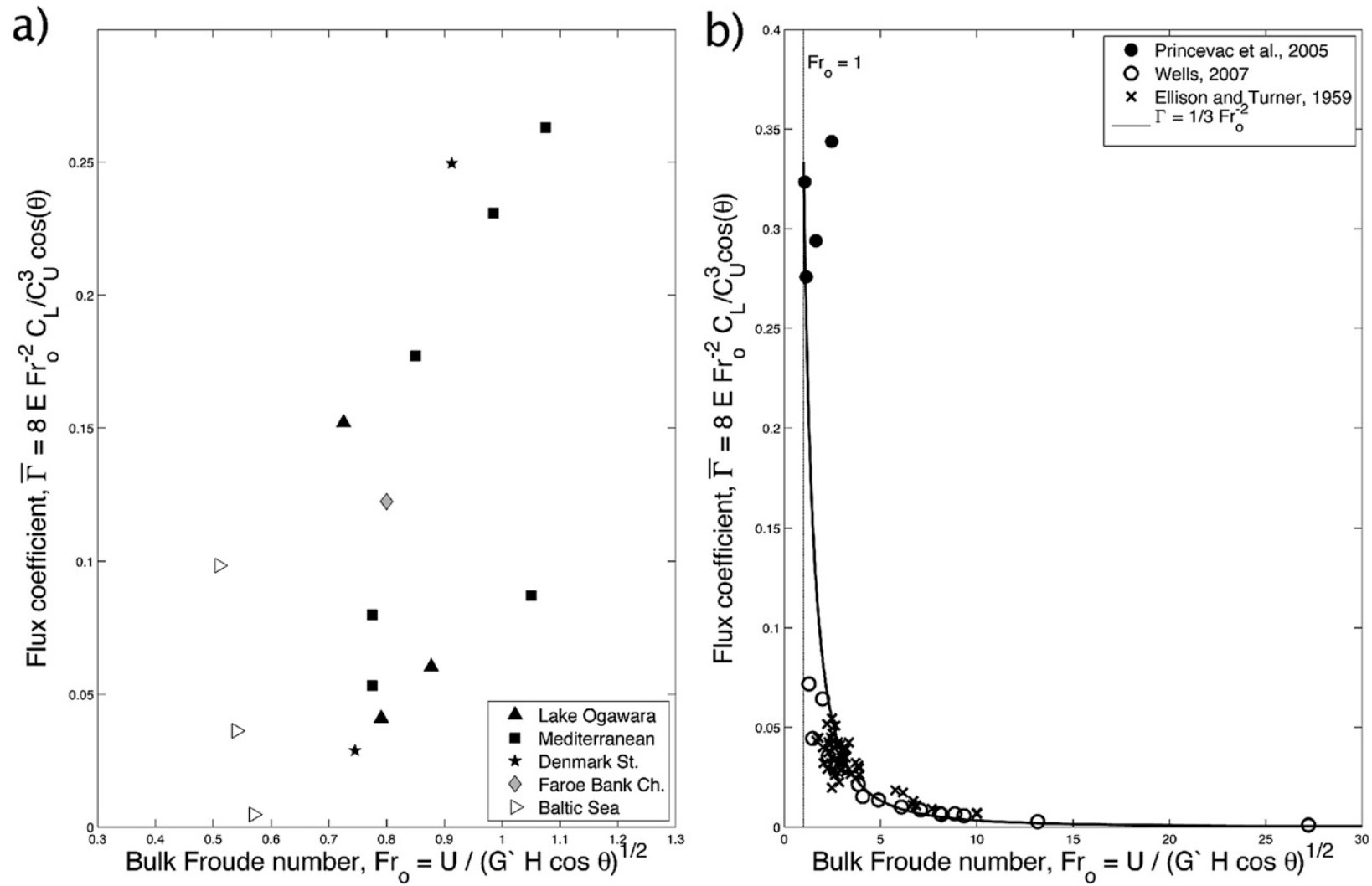

FIG. 8. (a) Using data of entrainment ratios from several sources shown in Fig. 2, (21), and $C_{U}^{3} / C_{L}=5 \times 10^{-2}$ to determine the inferred mixing efficiencies of the oceanographic density currents for $\operatorname{Fr}_{o}<1$. For this case, $\bar{\Gamma}$ is $O(0.1)$ and appears to increase with Froude number, consistent with the sketch in Fig. 3a. (b) The data from laboratory experiments where $\mathrm{Fr}_{o}<1$ and the katabatic current of Princevac et al. (2005) are plotted, assuming $C_{U}^{3} / C_{L} \sim 1$. The derived $\bar{\Gamma}$ compares well with the previously reported observation that $\bar{\Gamma} \sim \operatorname{Fr}_{o}^{n}$ for $\operatorname{Fr}_{o}>1$ : the vertical dashed line indicates $\operatorname{Fr}_{o}=1$.

an estimate of the entrainment ratio $E$. This estimate is plotted in Fig. 7a, and we note that the scaling laws that $E \sim \operatorname{Fr}_{T}^{2}$ for $\operatorname{Fr}_{T} \sim 1$ and $E \sim 0.1$ for $\operatorname{Fr}_{T} \gg 1$ are seen in this data. For comparison in Fig. 7b, we have replotted the data from Fig. 2 with the scaling laws $E \sim 0.025 \mathrm{Fr}_{o}^{2}$ and $E \sim 0.00025 \mathrm{Fr}_{o}^{2}$ suggested in the previous section.

We can also use the observation of entrainment ratios shown in Fig. 2 to estimate the flux coefficient of the various reported entraining density currents. We can determine $\bar{\Gamma}$ by rearranging Eq. (19) to give

$$
\bar{\Gamma}=8 C_{\epsilon} E \mathrm{Fr}_{o}^{-2} \cos \theta .
$$

Using (27) and the entrainment ratio data in Fig. 2, we can predict the flux coefficient $\bar{\Gamma}$, which we plot in Fig. 8a for oceanographic data where we assume a value of $C_{\epsilon}=$ $5 \times 10^{-2}$ for $\mathrm{Fr}<1$. The interesting result is that $\bar{\Gamma}$ is $O(0.1)$ as often assumed in oceanographic flows, but there is also a clear trend with $\bar{\Gamma}$ decreasing as the bulk Froude number decreases. Also there is some indication that $\bar{\Gamma}$ tends to zero for bulk Froude numbers of 0.5 , consistent with the discussion of Ivey and Imberger (1991) and Shih et al. (2005) that $\bar{\Gamma}$ goes to zero for a finite value of the turbulent Froude number, although the evidence is not conclusive because it is entirely possible that there remains some mixing at low Froude number, as predicted by the intermittent turbulence scaling of the previous section. The data in Fig. 8a shows good agreement with the conceptual sketch in Fig. 3a. This use of $C_{\epsilon}=5 \times 10^{-2}$ is an upper bound on this ratio and clearly using smaller $C_{\epsilon}$ would lead to higher estimates of $\bar{\Gamma}$.

For the laboratory data having $\mathrm{Fr}_{o}<1$ and the katabatic flow observations of Princevac et al. (2005), we assume $C_{\epsilon}=1$ and plot this as Fig. $8 \mathrm{~b}$ along with the curve $\bar{\Gamma}=(1 / 3) \operatorname{Fr}_{o}^{2}$ [as suggested by the data of Linden (1980), Ivey and Imberger (1991), and Strang and Fernando $(2001 \mathrm{a}, \mathrm{b})]$ and note that there is good agreement with this scaling for $\bar{\Gamma}$. It is noteworthy that the experimental data of Ellison and Turner (1959) and Wells (2007) appears to have a quite low flux coefficient. We have not plotted the data from Cenedese et al. (2004) and Cenedese and Adduce (2008) in this graph since we 
attribute the lower flux coefficient of these experimental flows to the decrease of mixing with Reynolds number, as shown in Fig. 5 and discussed in section 2b. The peak mixing efficiencies of $\bar{\Gamma}=0.25$ are found for the entrainment in katabatic winds reported by Princevac et al. (2005). It is somewhat surprising that this high Reynolds number flow appears to have a ratio $C_{\epsilon}$ similar to laboratory experiments rather than the oceanographic flows, and we hope that future work on the turbulence of katabatic flows can clarify if this is really the case.

\section{Conclusions}

The main result of this paper is the theoretical prediction that $E=(1 / 8) C_{\epsilon}\left(\bar{\Gamma} \mathrm{Fr}_{o}^{2} / \cos \theta\right)$, with $C_{\epsilon}$ as defined in (18). Based on the empirical dependence of $\bar{\Gamma}$ (and $C_{\epsilon}$ ) on $\mathrm{Fr}_{o}$, we predict three important scaling regimes: namely, that for large bulk Froude numbers we predict that $E$ is constant, consistent with observations of Turner (1986). For intermediate bulk Froude numbers, we predict that $E \sim \mathrm{Fr}_{o}^{2}$, and for small bulk Froude numbers $E$ will depend on $\mathrm{Fr}_{o}$ with a power law much greater than 2 . There is a paucity of data for the flux coefficient at low $\mathrm{Fr}_{o}$, and we hope that future studies may focus on this experimentally difficult regime that has great relevance for the ocean. This regime may be particularly important, as the recent work of Brethouwer et al. (2007) shows, that, provided the Reynolds number is sufficiently high, mixing events continue to occur even when the flow is very stratified (i.e., at asymptotically low bulk Froude number). At the moment, it is not really possible to distinguish whether the mixing is completely "switched off" or just markedly suppressed when stratification is very strong.

Also, the entrainment ratio observations of Cenedese et al. (2004) and Cenedese and Adduce (2008), which show an increase in entrainment ratio with Reynolds number, are consistent with the observations of Riley and de Bruyn Kops (2003) and Prastowo et al. (2008) that $\bar{\Gamma}$ increases with Reynolds number. This dependence of $E$ on Reynolds number partially explains why low Reynolds number laboratory experiments may have lower flux coefficients (and hence lower mixing efficiencies) than high Reynolds number density currents in the field.

Acknowledgments. Support for MGW was provided by NSERC, the Canadian Foundation for Innovation, the Ontario Research Fund, and the Connaught Committee of the University of Toronto. CPC gratefully acknowledges the hospitality and support of the 2008 Summer Study Program in Geophysical Fluid Dynamics at Woods Hole Oceanographic Institution, where this project was initiated.

\section{REFERENCES}

Arneborg, L., V. Fiekas, L. Umlauf, and H. Burchard, 2007: Gravity current dynamics and entrainment_-A process study based on observations in the Arkona Basin. J. Phys. Oceanogr., 37, 2094-2113.

Baines, P., 2001: Mixing in flows down gentle slopes into stratified environments. J. Fluid Mech., 443, 237-270.

Baringer, M. O., and J. F. Price, 1997: Mixing and spreading of the Mediterranean outflow. J. Phys. Oceanogr., 27, 1654-1677.

Billant, P., and J.-M. Chomaz, 2001: Self-similarity of strongly stratified inviscid flows. Phys. Fluids, 13, 1645-1651.

Brethouwer, G., P. Billant, E. Lindborg, and J.-M. Chomaz, 2007: Scaling analysis and simulation of strongly stratified turbulent flows. J. Fluid Mech., 585, 343-368.

Canuto, V. M., M. S. Dubovikov, and Y. Cheng, 2005: Entrainment: Local and non-local turbulence models with double diffusion. Geophys. Res. Lett., 32, L22604, doi:10.1029/2005GL023771.

Caulfield, C. P., W. Tang, and S. C. Plasting, 2004: Reynolds number dependence of an upper bound for the long-time-averaged buoyancy flux in plane stratified Couette flow. J. Fluid Mech., 498, 315-332.

Cenedese, C., and C. Adduce, 2008: Mixing in a density-driven current flowing down a slope in a rotating fluid. J. Fluid Mech., 604, 369-388.

$\longrightarrow$, and - 2010: A new parameterization for entrainment in overflows. J. Phys. Oceanogr., 40, 1835-1850.

_ J. A. Whitehead, T. A. Ascarelli, and M. Ohiwa, 2004: A dense current flowing down a sloping bottom in a rotating fluid. J. Phys. Oceanogr., 34, 188-203.

Christodoulou, G. C., 1986: Interfacial mixing in stratified flows. J. Hydraul. Res., 24, 77-92.

Crapper, P. F., and P. F. Linden, 1974: The structure of turbulent density interfaces. J. Fluid Mech., 65, 45-63.

Dallimore, C. J., J. Imberger, and T. Ishikawa, 2001: Entrainment and turbulence in saline underflow in Lake Ogawara. J. Hydraul. Eng., 127, 937-948.

Ellison, T. H., and J. S. Turner, 1959: Turbulent entrainment in stratified flows. J. Fluid Mech., 6, 423-448.

Fer, I., G. Voet, K. S. Seim, B. Rudels, and K. Latarius, 2010: Intense mixing of the Faroe Bank Channel overflow. Geophys. Res. Lett., 37, L02604, doi:10.1029/2009GL041924.

Fernando, H. J. S., 1991: Turbulent mixing in stratified fluids. Annu. Rev. Fluid Mech., 23, 455-493.

Garcia, M., 1990: Depositing and eroding turbidity sediment driven flows: Turbidity currents. Project Rep. 306, St. Anthony Falls Hydraulic Laboratory, University of Minnesota, Minneapolis, $179 \mathrm{pp}$.

Girton, J. B., and T. B. Sanford, 2003: Descent and modification of the overflow plume in Denmark Strait. J. Phys. Oceanogr., 33, 1351-1364.

Gregg, M. C., 1987: Diapycnal mixing in the thermocline: A review. J. Geophys. Res., 92, 5249-5286.

Holford, J. M., and P. F. Linden, 1999: Turbulent mixing in a stratified fluid. Dyn. Atmos. Oceans, 30, 173-198.

Huang, H., J. Imran, and C. Pirmez, 2007: Numerical modeling of poorly sorted depositional turbidity currents. J. Geophys. Res., 112, C01014, doi:10.1029/2006JC003778.

Itsweire, E. C., K. N. Helland, and C. W. van Atta, 1986: The evolution of grid-generated turbulence in a stably stratified fluid. J. Fluid Mech., 162, 299-338.

Ivey, G. N., and J. Imberger, 1991: On the nature of turbulence in a stratified fluid. Part I: The energetics of mixing. J. Phys. Oceanogr., 21, 650-659. 
_ - K. B. Winters, and J. R. Koseff, 2008: Density stratification, turbulence, but how much mixing? Annu. Rev. Fluid Mech., 40, 169-184.

Jackson, L., R. Hallberg, and S. Legg, 2008: A parameterization of shear-driven turbulence for ocean climate models. J. Phys. Oceanogr., 38, 1033-1053.

Johnson, G. C., R. G. Lueck, and T. B. Sanford, 1994: Stress on the Mediterranean outflow plume. Part II: Turbulent dissipation and shear measurements. J. Phys. Oceanogr., 24, 2084-2092.

Kato, H., and O. M. Phillips, 1969: On the penetration of the turbulent layer into a stratified fluid. J. Fluid Mech., 37, 643-665.

Legg, S., and Coauthors, 2009: Improving the oceanic overflow representation in climate models: The gravity current entrainment climate process team. Bull. Amer. Meteor. Soc., 90, 657-670.

Lienhard, J. H., and C. W. van Atta, 1990: The decay of turbulence in thermally stratified flow. J. Fluid Mech., 210, 57-112.

Linden, P. F., 1973: The interaction of a vortex ring with a sharp density interface: A model for turbulent entrainment. J. Fluid Mech., 60, 467-480.

_ 1979: Mixing in stratified fluids. Geophys. Astrophys. Fluid Dyn., 13, 3-23.

_ 1980 : Mixing across a density interface produced by grid turbulence. J. Fluid Mech., 100, 691-703.

List, E. J., 1982: Turbulent jets and plumes. Annu. Rev. Fluid Mech., 14, 189-212.

Martin, J. E., and C. R. Rehmann, 2006: Layering in a flow with diffusively stable temperature and salinity stratification. J. Phys. Oceanogr., 36, 1457-1470.

Mauritzen, C., J. F. Price, T. B. Sanford, and D. Torres, 2005: Circulation and mixing in the Faroese Channels. Deep-Sea Res. I, 52, 883-913.

Odier, P., J. Chen, M. K. Rivera, and R. E. Ecke, 2009: Fluid mixing in stratified gravity currents: The Prandtl mixing length. Phys. Rev. Lett., 102, 134504, doi:10.1103/PhysRevLett.102.134504.

Osborn, T. R., 1980: Estimates of the local rate of vertical diffusion from dissipation measurements. J. Phys. Oceanogr., 10, 83-89.

Papadakis, M. P., E. P. Chassignet, and R. W. Hallberg, 2003: Numerical simulations of the Mediterranean Sea outflow: Impact of the entrainment parameterization in an isopycnic coordinate ocean model. Ocean Modell., 5, 325-356.

Peltier, W. R., and C. P. Caulfield, 2003: Mixing efficiency in stratified shear flows. Annu. Rev. Fluid Mech., 35, 135-167.

Peters, H., and W. E. Johns, 2005: Mixing and entrainment in the Red Sea outflow plume. Part II: Turbulence characteristics. J. Phys. Oceanogr., 35, 584-600.

Prastowo, T., R. W. Griffiths, G. O. Hughes, and A. Hogg, 2008: Mixing efficiency in controlled exchange flows. J. Fluid Mech., 600, 235-244.

Price, J. F., and M. O. Barringer, 1994: Outflows and deep water production by marginal seas. Prog. Oceanogr., 33, 161-200.

Princevac, M., H. J. S. Fernando, and D. C. Whiteman, 2005: Turbulent entrainment into natural gravity-driven flows. J. Fluid Mech., 33, 259-268.

Rehmann, C. R., and J. R. Koseff, 2004: Mean potential energy change in stratified grid turbulence. Dyn. Atmos. Oceans, 37, 271-294.

Riley, J. J., and S. M. de Bruyn Kops, 2003: Dynamics of turbulence strongly influenced by buoyancy. Phys. Fluids, 15, 2047-2059.
— terpretation of some geophysical turbulence measurements. J. Atmos. Sci., 65, 2416-2424.

Rohr, J. J., E. C. Itsweire, K. N. Helland, and C. W. Van Atta, 1988: Growth and decay of turbulence in a stably stratified shear flow. J. Fluid Mech., 195, 77-111.

Sherwin, T. J., 2010: Observations of the velocity profile of a fast and deep oceanic density current constrained in a gully. J. Geophys. Res., 115, C03013, doi:10.1029/2009JC005557.

Shih, L. H., J. R. Koseff, G. N. Ivey, and J. H. Ferziger, 2005: Parameterization of turbulent fluxes and scales using homogeneous sheared stably stratified turbulence simulations. J. Fluid Mech., 525, 193-214.

Smyth, W. D., and J. N. Moum, 2000a: Length scales of turbulence in stably stratified mixing layers. Phys. Fluids, 12, 1327-1342.

, and $-2000 \mathrm{~b}$ : Anisotropy of turbulence in stably stratified mixing layers. Phys. Fluids, 12, 1343-1362.

— _ _ , and D. R. Caldwell, 2001: The efficiency of mixing in turbulent patches: Inferences from direct simulations and microstructure observations. J. Phys. Oceanogr., 31, 19691992.

Stillinger, D. C., K. N. Helland, and C. W. Van Atta, 1983: Experiments on the transition of homogeneous turbulence to internal waves in a stratified fluid. J. Fluid Mech., 131, 91-122.

Strang, E. J., and H. J. S. Fernando, 2001a: Entrainment and mixing in stratified shear flows. J. Fluid Mech., 428, 349-386.

-, and —, 2001b: Vertical mixing and transports through a stratified shear layer. J. Phys. Oceanogr., 31, 2026-2048.

Taylor, G. I., 1945: Dynamics of a mass of hot gas rising in air. U.S. Atomic Energy Commission, MDDC-919, LA Rep. 236, 19 pp. [Available online at http://www.fas.org/sgp/othergov/doe/ lanl/docs1/00407667.pdf.]

Turner, J. S., 1973: Buoyancy Effects in Fluids. Cambridge University Press, $367 \mathrm{pp}$.

, 1986: Turbulent entrainment: The development of the entrainment assumption, and its application to geophysical flows. J. Fluid Mech., 173, 431-471.

Umlauf, L., and L. Arneborg, 2009: Dynamics of rotating shallow gravity currents passing through a channel. Part I: Observation of transverse structure. J. Phys. Oceanogr., 39, 2385-2401.

Wells, M. G., 2007: Influence of Coriolis forces on turbidity currents and sediment deposition. Particle-Laden Flow: From Geophysical to Kolmogorov Scales, B. J. Geurts, H. Clercx, and W. Uijttewaal, Eds., ERCOFTAC Series, Vol. 11, Springer, 331-343.

-, and J. S. Wettlaufer, 2007: The long-term circulation driven by density currents in a two-layer stratified basin. J. Fluid Mech., 572, 37-58.

, and P. Nadarajah, 2009: The intrusion depth of density currents flowing into stratified water bodies. J. Phys. Oceanogr., 39, 1935-1947.

Xu, J. P., M. A. Noble, and L. K. Rosenfeld, 2004: In-situ measurements of velocity structure within turbidity currents. Geophys. Res. Lett., 31, L09311, doi:10.1029/2004GL019718.

Zellouf, Y., P. Dupont, and H. Peerhossaini, 2005: Heat and mass fluxes across density interfaces in a grid-generated turbulence. Int. J. Heat Mass Transfer, 48, 3722-3735. 